\title{
Diagnostica per immagini delle malattie del fegato e delle vie biliari
}

\author{
L. Grazioli • L. Olivetti
}

\author{
Masson Editore (2005) \\ ISBN 88-214-2729-3
}

Published online: 11 October 2006

Il nuovo libro si propone come un punto di riferimento nella diagnostica per immagini del fegato e delle vie biliari. L'articolazione dei capitoli prevede infatti un progressivo passaggio delle nozioni di base per arrivare alla parte fondamentale del libro dedicata agli aspetti semeiologici delle varie patologie del fegato e delle vie biliari.

A questo proposito il libro si divide in 8 parti e 25 capitoli. La prima parte è dedicata all'anatomia, dapprima normale, macro e microscopica, e successivamente radiologica, con particolare riferimento all'ecografia, alla TC e alla RM. Una attenzione particolare viene riservata all'anatomia vascolare e all'anatomia segmentaria del fegato.

La seconda parte è dedicata alla clinica delle malattie epatiche e delle vie biliari, con una ulteriore distinzione tra le malattie epatiche focali nel paziente epatopatico e non epatopatico. La terza parte entra nel merito delle tecniche di immagine e la relativa semeiotica ed è dedicata all'ecografia, alla TC, alla $R M$, alla Medicina Nucleare e alla Colangio-pancreatografia retrograda endoscopia e RM. In questa parte vengono inoltre trattati i mezzi di contrasto in TC e RM.

Nella quarta, quinta e sesta parte viene estesamente trattata la patologia epatica e biliare, con grande ricchezza sia di informazioni sia di iconografia.

Nella settima parte viene trattata la radiologia interventistica del fegato con particolare riguardo alla chemioembolizzazione e alle tecniche ablative percutanee dell'epatocarcinoma e delle metastasi epatiche, nonché alla TIPS e ai drenaggi biliari. L'ultima parte, infine, a testimoniare la completezza dell'opera, è dedicata al fegato trapiantato.

Il libro rappresenta un testo molto aggiornato e completo sulla diagnostica per immagini del fegato e delle vie biliari.

Riccardo Manfredi Istituto di Radiologia Policlinico G.B. Rossi

P.le L.A. Scuro

I-37134 Verona

e-mail: riccardo.manfredi@univr.it 